\title{
Notch signalling induces epithelial-mesenchymal transition to promote metastasis in oral squamous cell carcinoma
}

\author{
JIANPING ZHANG ${ }^{1}$, GENJIAN ZHENG ${ }^{2}$, LAN ZHOU ${ }^{2}$, PENGCHENG LI $^{2}$, MAN YUN ${ }^{2}$, \\ QI SHI ${ }^{1}$, TINGLI WANG ${ }^{1}$ and XIAOTONG WU ${ }^{1}$ \\ ${ }^{1}$ School of Clinical Medicine, Hainan Medical University, Haikou, Hainan 571199; \\ ${ }^{2}$ Department of Stomatology, The First Affiliated Hospital of Hainan Medical University, \\ Haikou, Hainan 570102, P.R. China
}

Received December 20, 2017; Accepted June 26, 2018

DOI: $10.3892 /$ ijmm.2018.3769

\begin{abstract}
The activation of Notch signalling induces epithelial-mesenchymal transition (EMT), but this signalling pathway and its association with EMT in the context of cell motility in oral squamous cell carcinoma (OSCC) remains unclear. The present study aimed to investigate the role of the Notch signalling pathway and EMT in the metastatic potential of OSCC using 2 cell lines, Tca8113 and CAL27. The data demonstrated that zinc finger domain SNAI1 (Snail) knockdown by small interfering RNA decreased the expression of vimentin and increased the expression of epithelial cadherin (E-cadherin). In addition, silencing Snail also significantly inhibited cell migration in the 2 OSCC cell lines. It was also identified that blocking Notch signalling with the g-secretase inhibitor DAPT decreased the expression of the EMT markers Snail and vimentin and increased E-cadherin expression, accompanied by a significant inhibition of cell migration in the 2 OSCC cell lines. These data clearly indicate that Notch signalling mediates EMT to promote metastasis in OSCC cells. Therefore, targeting Notch signalling and its association with EMT may provide novel insights into the mechanism of invasion and metastasis in OSCC and potential therapeutic interventions.
\end{abstract}

\section{Introduction}

Oral cavity cancer is one of the most common types of cancer worldwide, and oral squamous cell carcinoma (OSCC) is the most prevalent type of tumour at this site, accounting for $\sim 90 \%$ of all cases $(1,2)$. Despite previous advancements in diagnostic techniques and therapeutic strategies, the prognosis

Correspondence to: Professor Genjian Zheng, Department of Stomatology, The First Affiliated Hospital of Hainan Medical University, 31 Longhua Road, Haikou, Hainan 570102, P.R. China E-mail: airforcezhgj@163.com

Key words: oral squamous cell carcinoma, zinc finger domain SNAI1, epithelial-mesenchymal transition, Notch signalling, metastasis of OSCC has not improved, with an overall 5-year survival rate of $\sim 50 \%(2,3)$. This poor prognosis is primarily attributed to the high rates of invasion and metastasis (4). Therefore, it is important to understand the molecular events associated with tumour development and to identify biomarkers for prognosis assessment in OSCC.

Epithelial-mesenchymal transition (EMT) is a complex biological process that is important for embryogenesis, wound healing and malignant tumour metastasis (5). During EMT, epithelial tumour cells lose their polarity and adhesive phenotype, and they acquire mesenchymal traits, including an increase in cell motility potential and cytoskeletal remodelling (5). Alterations of adhesion molecules and extracellular proteins are key events in tumour cells during EMT. Epithelial cadherin (E-cadherin) and vimentin are two hallmarks of EMT; one is a predominant cell-surface protein for adherens junctions in epithelial cells, and the other is an intermediate filament in mesenchymal cells that controls cell motility, respectively (6). In primary head and neck squamous cell carcinoma cancer, the loss of E-cadherin and the gain of vimentin functions are associated with a higher incidence of distant metastasis (7). In addition, zinc finger protein SNAI1 (Snail) is one of the transcription factors involved in the EMT process and regulates various aspects of the EMT phenotype, including the upregulation of mesenchymal markers and the suppression of epithelial markers (6).

The Notch signalling pathway is evolutionarily conserved and serves important roles in numerous developmental processes, including cell fate determination, proliferation and differentiation (8). Notch signalling is initiated when a Notch ligand binds to an adjacent Notch receptor between two neighbouring cells. In mammals, the Notch family consists of 4 transmembrane receptors (Notch-1-4) and 5 ligands [Delta-like protein (Delta-like) 1, Delta-like 3, Delta-like 4, protein jagged (Jagged) 1 and Jagged-2] (9). Dysregulation of Notch signalling results in human developmental anomalies, including Alagille syndrome, cardiac disease and cancer development (10). Previous studies demonstrated that the expression levels of certain Notch receptors and ligands were dysregulated in human OSCC, which suggested that Notch signalling serves a vital role in tumour development in OSCC (11-13). 
Aberrant expression of EMT markers, including downregulated E-cadherin and $\beta$-catenin and upregulated vimentin and Neural cadherin, has been identified in OSCC tissues $(14,15)$. The promotion of EMT in OSCC involved signalling, including the phosphoinositide 3-kinase/protein kinase B and Wnt/ $\beta$-catenin signalling pathways (16). Notch signalling is also a key regulator in the induction of EMT, but the role of this signalling pathway and its association with EMT in cell motility in OSCC remains unclear. Therefore, the present study used a small-interfering RNA (siRNA) targeting Snail and a novel $\gamma$-secretase inhibitor, DAPT, to examine the role of the Notch signalling pathway and the EMT mechanism in the metastatic potential of OSCC in vitro. The Tca8113 and CAL27 human OSCC cell lines were adopted as cell models as they have been widely used to study the role of Notch signalling and EMT in cancer metastasis $(11,17,18)$. The results of the present study demonstrated that Snail-induced EMT may promote the migration of OSCC cells, and that Notch signalling may mediate tumour metastasis in OSCC cells via its association with EMT progression.

\section{Materials and methods}

Reagents. DAPT (GSI-IX) was purchased from Selleck Chemicals (Houston, TX, USA). Dulbecco's modified Eagle's medium (DMEM), RPMI-1640, foetal bovine serum (FBS) and trypsin-EDTA were supplied by Thermo Fisher Scientific, Inc. (Waltham, MA, USA). Primary rabbit anti-human Notch1 intracellular domain (N1ICD; cat. no., ab83232) monoclonal antibodies were obtained from Abcam (Cambridge, MA, USA). The rabbit anti-human Snail (cat. no., 3879), E-cadherin (cat. no., 3195), and vimentin (cat. no., 5741) antibodies and the GAPDH (cat. no., 5174) control antibody were all acquired from Cell Signaling Technology (Danvers, MA, USA). The horseradish peroxidase-labelled secondary antibody (cat. no., EK020) was obtained from Xi'an Zhuangzhi Biotech Co., Ltd. (Xi'an, China).

Cell cultures. Established Tca8113 and CAL27 human OSCC cell lines were purchased from CHI Scientific, Ltd., (Wuxi, China). TCA8113 cells were maintained in RPMI-1640 medium containing $20 \% \mathrm{FBS}, 100 \mathrm{U} / \mathrm{ml}$ penicillin $\mathrm{G}$ and $100 \mu \mathrm{g} / \mathrm{ml}$ streptomycin, while CAL27 cells were maintained in DMEM containing 10\% FBS, $100 \mathrm{U} / \mathrm{ml}$ penicillin $\mathrm{G}$ and $100 \mu \mathrm{g} / \mathrm{ml}$ streptomycin. The 2 cell lines were incubated in a humidified atmosphere of $5 \% \mathrm{CO}_{2}$ at $37^{\circ} \mathrm{C}$. Cells in the logarithmic growth phase were used in subsequent experiments.

Cell viability assay. $5 \times 10^{3}$ Tca 8113 or CAL27 cells were seeded in 96-well plates for $24 \mathrm{~h}$, then cells were treated with 6 dilutions of DAPT in DMSO $(1,2,5,10,20$ and $40 \mu \mathrm{mol} / \mathrm{l})$ for 24,48 and $72 \mathrm{~h}$, and cell viability was determined by an MTT assay. Following DAPT exposure, the medium was replaced with fresh medium containing $20 \mu \mathrm{l}(5 \mathrm{mg} / \mathrm{ml}$ PBS $)$ MTT. After $4 \mathrm{~h}$ of incubation at $37^{\circ} \mathrm{C}$, the medium was removed, and $150 \mu \mathrm{l}$ DMSO was added to dissolve the formazan. Absorbance measurements were made with an automatic ELISA reader (Infinite M200; Tecan Group, Ltd., Männedorf, Switzerland) at $490 \mathrm{~nm}$. Each experiment included 6 replicates and was repeated 3 times.
Transient transfection of Snail siRNA. Viral transfer vectors encoding green fluorescent protein and Snail siRNA, negative control (NC) siRNA, polybrene and Enhanced Infection Solution (ENi.S) were constructed by Shanghai Genechem Co., Ltd. (Shanghai, China). The target sequence for the Snail siRNA was as follows: 5'-AGGACTCTAATCCAGAGTT-3'. Prior to viral infection, Tca8113 and CAL27 cells were seeded into 6 -well plates at a density of $5 \times 10^{4}$ cells/well. Following $24 \mathrm{~h}$ of incubation at $37^{\circ} \mathrm{C}$, the cells were transiently transfected with Snail siRNA or NC siRNA using viral transfer vectors when they reached $40 \%$ confluence. Cell transfection was performed according to the protocol of the manufacturer of the transfection kit. The CAL27 virus infection solution contained $20 \mu \mathrm{l}$ virus at titer of $1 \mathrm{E}+8 \mathrm{TU} / \mathrm{ml}, 880 \mu \mathrm{l} \mathrm{ENi} . \mathrm{S}$ and $100 \mu \mathrm{l}$ phycoerythrin (enriched with $50 \mu \mathrm{g} / \mathrm{ml}$ polybrene ENi.S solution), and the Tca8113 virus infection solution contained $120 \mu \mathrm{l}$ virus at titer of $1 \mathrm{E}+8 \mathrm{TU} / \mathrm{ml}, 780 \mu \mathrm{l} \mathrm{ENi} . \mathrm{S}$ and $100 \mu \mathrm{l}$ complete medium containing $50 \mu \mathrm{g} / \mathrm{ml}$ polybrene. After $12 \mathrm{~h}$ of incubation at $37^{\circ} \mathrm{C}$, the virus infection solution was replaced with conventional culture medium to maintain cell growth for $96 \mathrm{~h}$ prior to the subsequent experiments.

Migration and wound-healing assays. Cellular Transwell migration was assayed by non-Matrigel-coated Transwell cell culture chambers (8- $\mu \mathrm{m}$ pore size; Corning Incorporated, Corning, NY, USA). Briefly, $1 \times 10^{5}$ Tca 8113 or CAL27 cells were seeded into the upper chamber in $200 \mu 1$ serum-free medium, and $600 \mu \mathrm{l}$ complete culture medium with $10 \%$ FBS for each cell line was placed in the lower chamber as a chemoattractant. Cell migration assays following Snail knockdown were conducted at $37^{\circ} \mathrm{C}$ for $24 \mathrm{~h}$, and subsequent to treatment with DAPT, the assays were conducted at $37^{\circ} \mathrm{C}$ for 24 and $48 \mathrm{~h}$. Then, at room temperature, the cells were fixed with $4 \%$ paraformaldehyde for $10 \mathrm{~min}$ and stained with $0.1 \%$ crystal violet for $20 \mathrm{~min}$. The unmigrated cells were removed from the upper surface of the membrane using cotton-tipped swabs. Finally, the migrated cells were counted at magnification, $x 400$ from 9 different randomly selected fields of each filter using an inverted light microscope.

For the wound-healing assays, $5 \times 10^{5} \mathrm{Tca} 8113$ and CAL27 cells were seeded in 6-well plates in the aforementioned culture medium. When the cells reached $90 \%$ confluence, wounds were made by scratching a line across the bottom of the dish on a monolayer of confluent cells with a $10 \mu \mathrm{l}$ pipette tip. Then, the cells were incubated in serum-free media at $37^{\circ} \mathrm{C}$. The gaps between the cells at 0,24 and $48 \mathrm{~h}$ were imaged on a microscope at a magnification, $x 100$ and then quantified using ImageJ 1.8.0 software (National Institutes of Health, Bethesda, MD, USA).

Real time-quantitative polymerase chain reaction ( $R T-q P C R)$. The total RNA from all experimental and control cells was extracted using TRIzol ${ }^{\circledR}$ (Life Technologies; Thermo Fisher Scientific, Inc.) according to the manufacturer's protocol. Reverse transcription was performed using the PrimeScript RT Master Mix (Takara Bio, Inc., Otsu, Japan). The RT-qPCR was performed using a CFX96 real-time PCR detection system with SYBR ${ }^{\circledR}$ Premix Ex Taq II (Takara Bio, Inc.). Human GAPDH was used as an internal control to normalize the amount of mRNA in each sample using the $2^{-\Delta \Delta C q}$ 

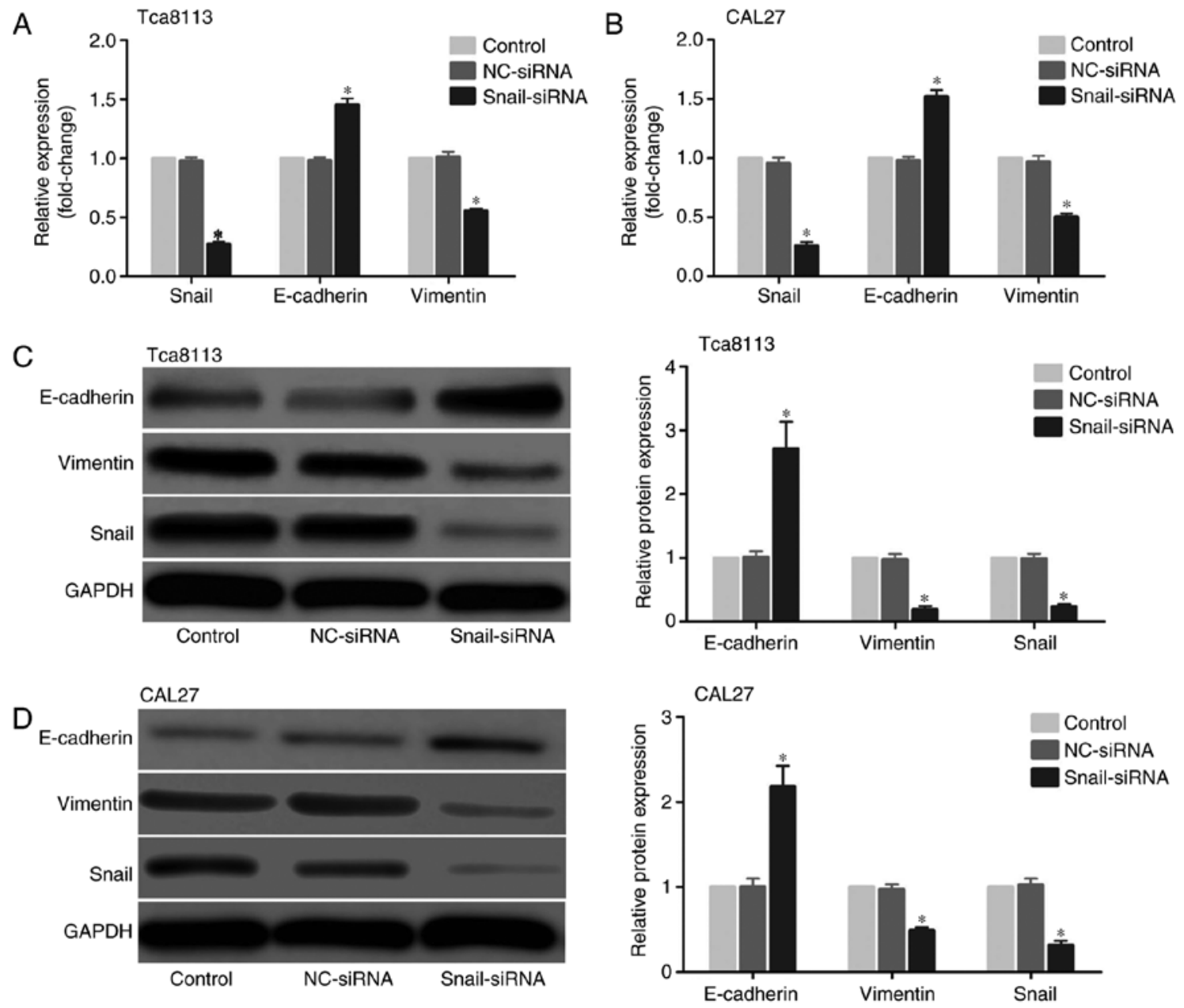

Figure 1. Downregulation of Snail decreases the epithelial-mesenchymal transition progression of oral squamous cell carcinoma cells. Tca8113 and CAL27 cells were transfected with NC siRNA and Snail siRNA; non-transfected cells served as controls. The expression of Snail, E-cadherin and vimentin at the mRNA level was measured by reverse transcription quantitative polymerase chain reaction in (A) Tca8113 and (B) CAL27 cells. (C) The expression of E-cadherin, vimentin and Snail at the protein level was measured by western blotting. (D) Densitometric analysis of data from (C). All data are presented as the mean \pm standard deviation from triplicate experiments. "P<0.05 vs. control and NC groups. siRNA, small interfering RNA; NC siRNA, negative control siRNA; Snail, zinc finger protein SNAI1; E-cadherin, epithelial cadherin.

method (19). The conditions for PCR were as follows: 1 cycle of $95^{\circ} \mathrm{C}$ for $30 \mathrm{sec}$, followed by 40 cycles of $95^{\circ} \mathrm{C}$ for $5 \mathrm{sec}$ and $60^{\circ} \mathrm{C}$ for $30 \mathrm{sec}$. The primers used in the PCR reactions were as follows: Vimentin forward (F), 5'-AACCTGGCCGAG GACATCA-3'; vimentin reverse (R), 5'-TCAAGGTCAAGA CGTGCCAGA-3'; Snail F, 5'-GACCACTATGCCGCGCTC TT-3'; Snail R, 5'-TCGCTGTAGTTAGGCTTCCGATT-3'; hes family bHLH transcription factor 1 (Hes1) F, 5'-GTGTCA ACACGACACCGGATAAAC-3'; Hes1 R, 5'-CAGAATGTC CGCCTTCTCCAG-3'; E-cadherin F, 5'-AGGATGACACCC GGGACAAC-3'; E-cadherin R, 5'-TGCAGCTGGCTCAAG TCAAAG-3'; GAPDH F, 5'-GCACCGTCAAGGCTGAGA AC-3'; GAPDH R, 5'-TGGTGAAGACGCCAGTGGA-3'.

Western blot analysis. The proteins were prepared using RIPA lysis buffer (Thermo Fisher Scientific, Inc.), and protein concentrations were determined using a BCA protein assay kit (Beyotime Institute of Biotechnology, Haimen, China). Each lane was loaded with $30 \mu \mathrm{g}$ protein on $6-10 \%$ SDS-PAGE gels and then transferred onto polyvinyl difluoride membranes (EMD Millipore, Billerica, MA, USA), which were blocked with $5 \%$ non-fat dry milk/TBST (0.1\% Tween-20) for $1 \mathrm{~h}$ at $25^{\circ} \mathrm{C}$ to prevent non-specific binding. The membranes were then incubated with primary antibodies (dilution, 1:1,000) in $5 \% \mathrm{w} / \mathrm{v}$ BSA, $1 \mathrm{X}$ TBS and $0.1 \%$ Tween -20 at $4{ }^{\circ} \mathrm{C}$ with gentle shaking overnight. Following washing 3 times with TBST buffer (20 mM Tris- $\mathrm{HCl}, 137 \mathrm{mM} \mathrm{NaCl}$ and $0.1 \%$ Tween-20; $\mathrm{pH} 7.6$ ) for $5 \mathrm{~min} /$ wash, the blots were incubated with a horseradish peroxidase-conjugated goat anti-rabbit secondary antibody $(1: 5,000)$ for $1 \mathrm{~h}$ at room temperature. Subsequently, the membrane was washed 3 times with TBST for $5 \mathrm{~min} /$ wash, and the protein bands were detected with the Millipore ECL luminous liquid (EMD Millipore). Image J 1.8.0 software was used to quantify the immunoblot bands.

Statistical analysis. Each experiment was repeated at least 3 times. All data are expressed as the mean \pm standard deviation and calculated by SPSS 19.0 software (SPSS, Inc., IBM Corp., Armonk, NY, USA). The one-way analysis of variance followed by Fisher's Least Significant Difference test were used to compare the differences among multiple groups. $\mathrm{P}<0.05$ was considered to indicate a statistically significant difference. 

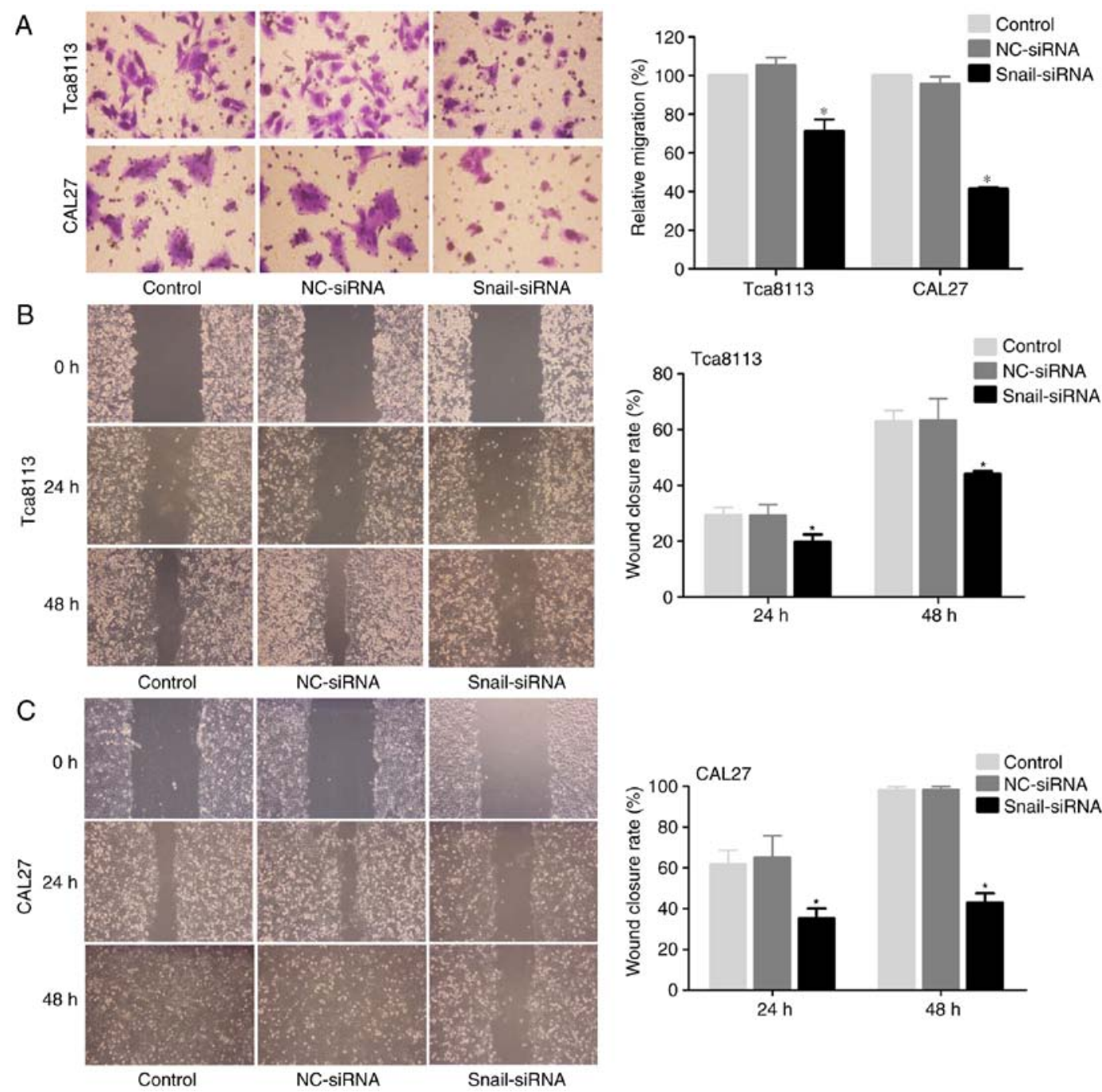

Figure 2. Snail silencing decreases the motility of oral squamous cell carcinoma cells. The migration of Snail-silenced Tca8113 and CAL27 cells was measured by a (A) Transwell assay for $24 \mathrm{~h}$ and a wound-healing assay for (B) 24 and (C) $48 \mathrm{~h}$. All data are presented as the mean \pm standard deviation from triplicate experiments. " $\mathrm{P}<0.05$ vs. control and NC group. Snail, zinc finger protein SNAI1; siRNA, small interfering RNA; NC siRNA, negative control siRNA.

\section{Results}

EMT participates in the migration of OSCC cells. To identify the role of EMT progression in the migration of OSCC cells, an siRNA method was used to knockdown Snail, a vital EMT transcriptional factor, in the OSCC Tca8113 and CAL27 cell lines. The mRNA level was first assessed using RT-qPCR, and the results demonstrated that the Snail siRNA model effectively suppressed Snail expression in OSCC cells. In addition, the expression level of the mesenchymal marker vimentin was decreased, while the level of the epithelial marker E-cadherin was increased in the Tca8113 and CAL27 cells (Fig. 1A and B). Then, changes in protein expression were examined with western blot analysis. In accordance with the mRNA results, Snail and vimentin levels were decreased, while E-cadherin was increased in the Tca8113 and CAL27 cells (Fig. 1C and D).

To assess the effect of Snail knockdown on cell migration in Tca8113 and CAL27 cells, a Transwell assay indicated that Snail-silenced cells exhibited a significant decrease in migration rate compared with the controls (Fig. 2A).
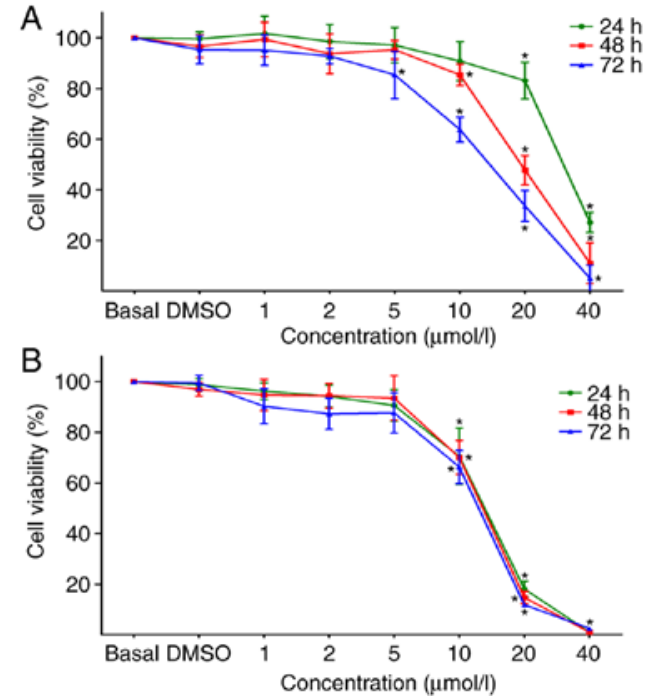

Figure 3. Effects of DAPT on the viability of oral squamous cell carcinoma cells. (A) Tca8113 and (B) CAL27 cells were treated with DAPT at different doses for 3 days. The viability of Tca8113 and CAL27 cells was measured by an MTT assay. The basal group (without any treatment) was used as control. All data are presented as the mean \pm standard deviation from triplicate experiments. ${ }^{*}<0.05$ vs. basal group. 

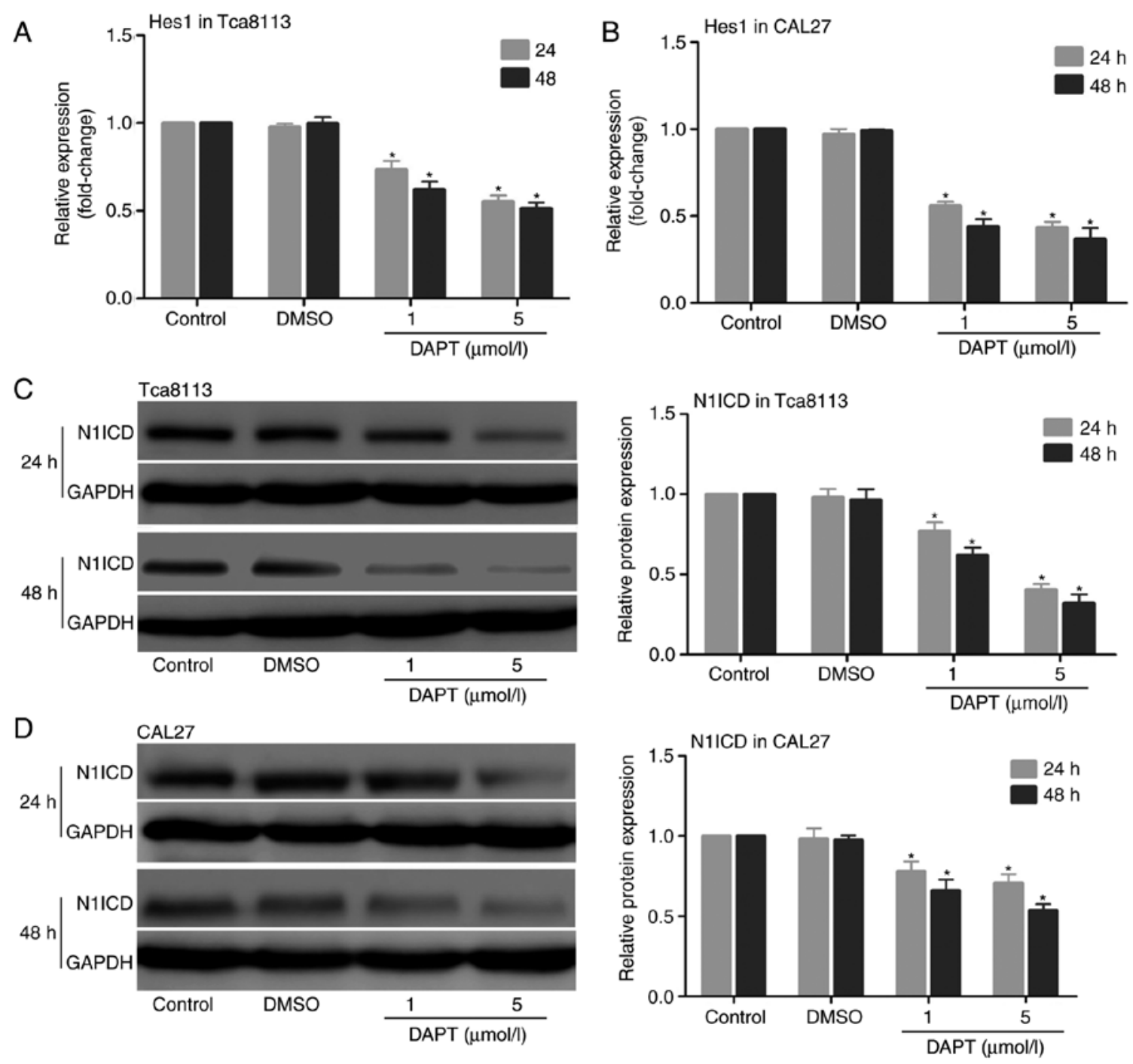

Figure 4. DAPT significantly inhibits the Notch signalling pathway in OSCC cells. (A) Tca8113 and (B) CAL27 cells were treated with DAPT at 1 and $5 \mu$ mol/1 for 24 and $48 \mathrm{~h}$. Untreated cells or cells treated with DMSO served as controls. The expression of Hes1 at the mRNA level was measured by reverse transcription quantitative polymerase chain reaction. The expression of N1ICD at the protein level in (C) Tca8113 and (D) CAL27 cells was measured by western blot analysis. All data are presented as the mean \pm standard deviation from triplicate experiments. ${ }^{*} \mathrm{P}<0.05$ vs. control group. DMSO, dimethyl sulfoxide; Hes1, hes family bHLH transcription factor 1; N1ICD, Notch1 intracellular domain.

Furthermore, it was observed that Snail knockdown in OSCC cells significantly decreased the rate of cell migration from the edge of the wound following scratching (Fig. 2B and C).

Inhibition of Notch signalling with DAPT inhibits EMT progression. To confirm the association between Notch signalling and EMT progression, the $\gamma$-secretase DAPT was employed to block Notch signalling and detect changes in the EMT mechanism in OSCC cells. Firstly, an MTT assay was used to examine the effects of DAPT at different doses $(1,2,5,10,20$ and $40 \mu \mathrm{mol} / \mathrm{l})$ on the growth and viability of Tca8113 and CAL27 cells, and untreated cells or cells treated with DMSO were used as controls. The results demonstrated that the viabilities of Tca8113 and CAL27 cells were markedly inhibited by DAPT in a time- and concentration-dependent manner when the concentrations of DAPT were $\geq 10 \mu \mathrm{mol} / \mathrm{l}$ (Fig. 3). Therefore, treatment with DAPT at concentrations of 1 and $5 \mu \mathrm{mol} / 1$ for 24 and $48 \mathrm{~h}$ was used in subsequent studies, which had no marked effect on the viability of Tca8113 and CAL27 cells.
Then, the role of DAPT in the expression of the Notch signalling pathway was examined in Tca8113 and CAL27 cells. The cells were treated with DAPT for 24 and $48 \mathrm{~h}$, and the mRNA expression levels of Hes1 and the protein expression levels of N1ICD were measured. The results indicated that the mRNA expression levels of Hesl and the protein expression levels of N1ICD were decreased in the 2 cell lines following treatment with DAPT in a time- and concentration-dependent manner (Fig. 4). Whether inhibition of the Notch signalling pathway with DAPT affected the expression of EMT markers in Tca8113 and CAL27 cells was also explored. The expression of Snail, vimentin and E-cadherin were detected at the mRNA and protein levels in the 2 cell lines. The results demonstrated that the inhibition of the Notch signalling pathway with DAPT downregulated the expression of Snail and vimentin and upregulated the expression of E-cadherin at the mRNA (Fig. 5A and B) and protein levels (Fig. 5C and D) in a time- and concentration-dependent manner. 
A
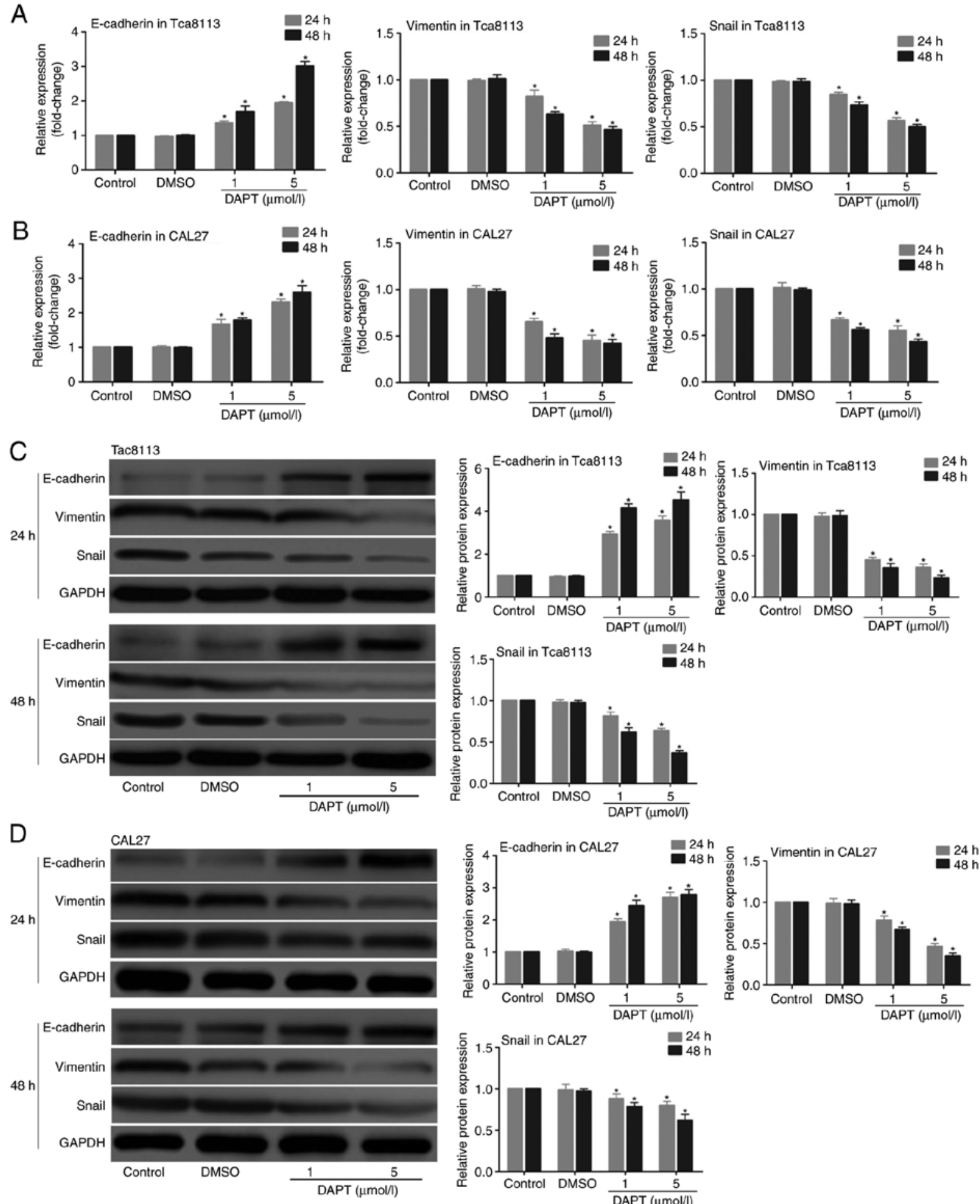

Figure 5. Inhibition of the Notch signalling pathway by DAPT decreases the epithelial-mesenchymal transition progression of oral squamous cell carcinoma cells. Tca8113 and CAL27 cells were treated with DAPT at 1 and $5 \mu \mathrm{mol} / 1$ for 24 and $48 \mathrm{~h}$. Untreated cells or cells treated with DMSO served as controls. The expression of E-cadherin, vimentin and Snail at the mRNA level was measured by reverse transcription-quantitative polymerase chain reaction in (A) Tca8113 and (B) CAL27 cells. The expression of E-cadherin, vimentin and Snail at the protein level was measured by western blot analysis in (C) Tca8113 and (D) CAL27 cells. All data are presented as the mean \pm standard deviation from triplicate experiments. "P $<0.05$ vs. control group. E-cadherin, epithelial cadherin; Snail, zinc finger domain SNAI1; DMSO, dimethyl sulfoxide.

Inhibition of Notch signalling with DAPT decreases OSCC cell migration. To determine whether Notch inhibition affects the migration potential of OSCC cells, Transwell chamber migration and wound-healing assays were performed. As indicated in Fig. 6A-D, the inhibition of Notch signalling with DAPT markedly decreased the migratory capabilities of Tca8113 and CAL27 cells in a time- and concentration-dependent manner, and the number of migrating cells was significantly decreased in the DAPT treatment groups compared with in the controls. 

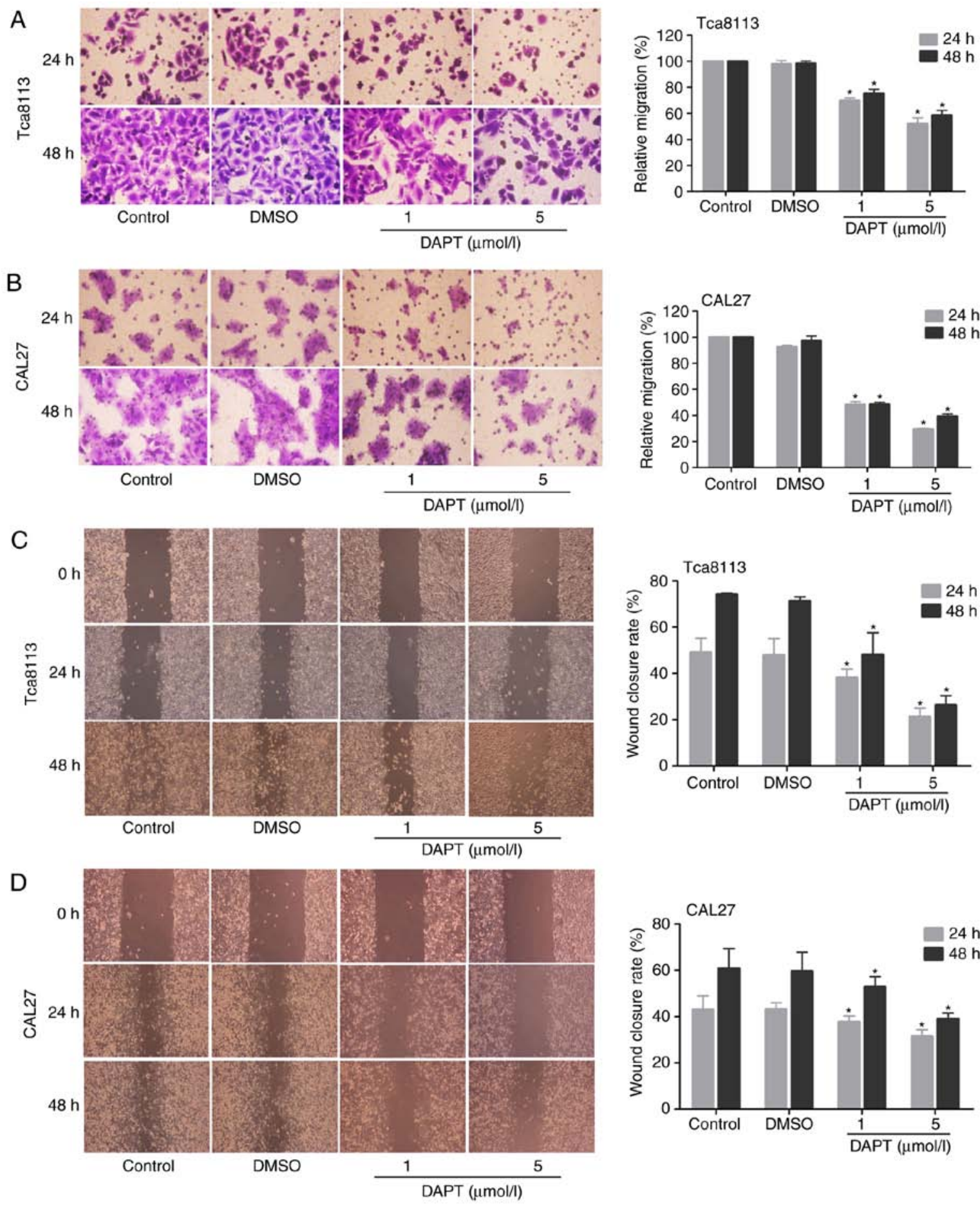

Figure 6. Inhibition of the Notch signalling pathway by DAPT decreases the motility of OSCC cells. Tca8113 and CAL27 cells were treated with DAPT at 1 and $5 \mu \mathrm{mol} / \mathrm{l}$ for 24 and $48 \mathrm{~h}$. Untreated cells or cells treated with DMSO cells served as controls. The migration of DAPT-treated OSCC cells was measured by a Transwell assay in (A) Tca8113 and (B) CAL27 cells and a wound-healing assay (C) Tca8113 and (D) CAL27 cells. All data are presented as the mean \pm standard deviation from triplicate experiments. * $\mathrm{P}<0.05$ vs. control group. DMSO, dimethyl sulfoxide; OSCC, oral squamous cell carcinoma.

\section{Discussion}

The high rate of distant metastasis is a major obstacle in improving the survival rates of patients with OSCC (20). Therefore, an understanding of the molecular mechanisms that regulate metastasis may provide novel insights for the development of effective treatments for OSCC, which in turn may decrease the rate of metastasis and improve survival. The present study aimed to understand the role of Notch signal- ling and its association with EMT in metastasis during OSCC development using 2 cell lines, Tca8113 and CAL27. Although STR detection identified that the Tca8113 cell line was contaminated by HeLa cells (21), previous studies revealed that contamination is unlikely to affect the outcomes of the present study $(13,17,22)$. Firstly, the results verified that Snail regulates EMT and promotes tumour migration in OSCC cells, and then it was demonstrated that the inhibition of Notch signalling by DAPT decreased the migration of OSCC cells 
by inhibiting the EMT mechanism. The present study suggests that Notch signalling promotes EMT and is involved in tumour metastasis in OSCC cells.

EMT is a dynamic cellular process during which epithelial cells adopt characteristics of mesenchymal cells. EMT itself is essential for tissue construction during normal development and the development of metastatic disease (5). A number of in vitro and in vivo studies have demonstrated that the cancer-associated EMT process promotes cancer cell migration and invasion into the stroma (23). A loss of epithelial markers and cell-cell adhesion, and a gain of mesenchymal marker expression, are key events in EMT for tumour cell metastasis and invasion (24). In previous studies, the downregulation of E-cadherin and matrix metalloproteinase (MMP)-9 and the upregulation of vimentin and MMP-2 were observed in OSCC cell lines (16), and a trend towards decreased E-cadherin expression but increased vimentin expression was associated with increased disease severity of OSCC (14). In addition, EMT markers, including E-cadherin, $\beta$-catenin, adenomatous polyposis coli and vimentin, have predictive value for the progression of multiple primary OSCC, and the simultaneous downregulation of E-cadherin and $\beta$-catenin exerted a significant prognostic effect in these patients (25). These data, combined with those from the present study, suggest that the EMT process serves a critical role in the progression of OSCC.

Snail is known as a pivotal mediator of EMT and contributes to the repression of the transcription of E-cadherin gene by binding to E-boxes during tumour progression (26). A number of studies have demonstrated that Snail promotes tumour development and metastasis through regulating EMT in a variety of types of cancer (27). A previous study demonstrated that Snail knockdown in OSCC cells significantly inhibited cell migration and invasion (28). An additional study suggested that the overexpression of Snail induces EMT and promotes cancer stem cell-like traits in OSCC SCC-9 cells (29). The results of the present study additionally confirmed that the knockdown of Snail upregulated E-cadherin expression, downregulated vimentin expression and decreased cell motility in Tca8113 and CAL27 cells. All of these data suggest that Snail-induced EMT promotes tumour metastasis in OSCC cells.

It is well-established that the EMT process is stimulated and regulated by a number of signal transduction pathways, including Transforming growth factor $\beta$, Wnt, Hedgehog and Notch signalling (30). Emerging evidence suggests that the Notch signalling pathway serves a vital role in the regulation of EMT, resulting in tumour invasion and metastasis $(31,32)$, and the suppression of Notch signalling with the $\gamma$-secretase inhibitor DAPT restricts the growth, invasion and metastasis of gastric cancer by inhibiting EMT (33). Previous immunohistochemical examination demonstrated increased expression levels of Notch1, Notch2, Jagged1, Hes1 and Hairy enhancer-of-split related with YRPW motif protein 1 in oral tissues of OSCC compared with those in the normal controls, suggesting that Notch signalling is active in OSCC (13). However, the roles of the Notch signalling pathway and the EMT process in the metastatic potential of OSCC remains unclear. In the present study, DAPT was used to inhibit the Notch signalling pathway in Tca8113 and CAL27 cells. The results indicated that the mRNA expression level of Hes1, a downstream target of Notch signalling, and the protein expression level of the Notch1 intracellular domain (N1ICD) were decreased following treatment with DAPT. These data suggest that DAPT effectively inhibits the Notch pathway in OSCC cell lines. In addition, the results suggested that the inhibition of Notch signalling notably decreased Snail and vimentin and increased E-cadherin at the mRNA and protein levels during the EMT process. Furthermore, DAPT inhibition may markedly decrease the migration ability of OSCC cells. Together, these observations indicate that Notch signalling may be involved in the EMT-induced metastasis of OSCC cells.

In summary, the results from the present study confirmed that Snail-induced EMT promotes cell migration in OSCC cells, and additionally demonstrated that Notch signalling mediates tumour metastasis in OSCC cells through its association with EMT progression. Therefore, targeting Notch signalling and its association with EMT may provide novel insights into the mechanism of invasion and metastasis in OSCC, and the inactivation of Notch signalling or the inhibition of the EMT upstream molecule Snail may be useful approaches for the development of therapeutic strategies for OSCC.

\section{Acknowledgements}

Not applicable.

\section{Funding}

The present study was supported by the Key Research and Development Program of Hainan Province (grant no., ZDYF 2016113).

\section{Availability of data and materials}

The datasets used and/or analyzed during the current study are available from the corresponding author on reasonable request.

\section{Authors' contributions}

JZ, GZ, QS and MY contributed to the study concept and experimental design. JZ, LZ, QS, XW, PL and TW performed the experiments. JZ, PL and TW contributed to the data acquisition and analysis. JZ and MY drafted and revised the manuscript. All authors read and approved the final manuscript.

\section{Ethics approval and consent to participate}

Not applicable.

\section{Patient consent for publication}

Not applicable.

\section{Competing interests}

The authors declare that they have no competing interests. 


\section{References}

1. Torre LA, Bray F, Siegel RL, Ferlay J, Lortet-Tieulent J and Jemal A: Global cancer statistics, 2012. CA Cancer J Clin 65: 87-108, 2015

2. Warnakulasuriya S: Living with oral cancer: Epidemiology with particular reference to prevalence and life-style changes that influence survival. Oral Oncol 46: 407-410, 2010.

3. Gupta S, Kong W, Peng Y, Miao Q and Mackillop WJ: Temporal trends in the incidence and survival of cancers of the upper aerodigestive tract in Ontario and the United States. Int J Cancer 125: 2159-2165, 2009.

4. Kim SY, Nam SY, Choi SH, Cho KJ and Roh JL: Prognostic value of lymph node density in node-positive patients with oral squamous cell carcinoma. Ann Surg Oncol 18: 2310-2317, 2011.

5. Li L and Li W: Epithelial-mesenchymal transition in human cancer: Comprehensive reprogramming of metabolism, epigenetics, and differentiation. Pharmacol Ther 150: 33-46, 2015.

6. Scanlon CS, Van Tubergen EA, Inglehart RC and D'Silva NJ: Biomarkers of epithelial-mesenchymal transition in squamous cell carcinoma. J Dent Res 92: 114-121, 2013.

7. Nijkamp MM, Span PN, Hoogsteen IJ, van der Kogel AJ, Kaanders $\mathrm{JH}$ and Bussink $\mathrm{J}$ : Expression of E-cadherin and vimentin correlates with metastasis formation in head and neck squamous cell carcinoma patients. Radiother Oncol 99: 344-348, 2011.

8. Artavanis-Tsakonas S, Rand MD and Lake RJ: Notch signaling: Cell fate control and signal integration in development. Science 284: 770-776, 1999.

9. Yamamoto S, Schulze KL and Bellen HJ: Introduction to Notch signaling. Methods Mol Biol 1187: 1-14, 2014.

10. Bray SJ: Notch signalling: A simple pathway becomes complex. Nat Rev Mol Cell Biol 7: 678-689, 2006.

11. Zhang TH, Liu HC, Zhu LJ, Chu M, Liang YJ, Liang LZ and Liao GQ: Activation of Notch signaling in human tongue carcinoma. J Oral Pathol Med 40: 37-45, 2011.

12. Osathanon T, Nowwarote N and Pavasant P: Expression and influence of Notch signaling in oral squamous cell carcinoma. J Oral Sci 58: 283-294, 2016.

13. Hijioka H, Setoguchi T, Miyawaki A, Gao H, Ishida T, Komiya S and Nakamura N: Upregulation of Notch pathway molecules in oral squamous cell carcinoma. Int J Oncol 36: 817-822, 2010.

14. Chaw SY, Majeed AA, Dalley AJ, Chan A, Stein S and Farah CS: Epithelial to mesenchymal transition (EMT) biomarkers-E-cadherin, beta-catenin, APC and Vimentin - in oral squamous cell carcinogenesis and transformation. Oral Oncol 48: 997-1006, 2012.

15. Angadi PV, Patil PV, Angadi V, Mane D, Shekar S, Hallikerimath S, Kale AD and Kardesai SG: Immunoexpression of epithelial mesenchymal transition proteins E-cadherin, $\beta$-catenin, and $\mathrm{N}$-cadherin in oral squamous cell carcinoma. Int J Surg Pathol 24: 696-703, 2016.

16. Krisanaprakornkit S and Iamaroon A: Epithelial-mesenchymal transition in oral squamous cell carcinoma. ISRN Oncol 2012: 681469, 2012.
17. Xie SM, Lu ZY, Lin YZ, Shen LJ and Yin C: Upregulation of PTEN suppresses invasion in Tca8113 tongue cancer cells through repression of epithelial-mesenchymal transition (EMT). Tumor Biol 37: 6681-6689, 2016.

18. Zhao XP, Zhang H, Jiao JY, Tang DX, Wu YL and Pan CB: Overexpression of $H M G A 2$ promotes tongue cancer metastasis through EMT pathway. J Transl Med 14: 26, 2016.

19. Livak KJ and Schmittgen TD: Analysis of relative gene expression data using real-time quantitative PCR and the $2^{-\Delta \Delta C_{\mathrm{T}}}$ method. Methods 25: 402-408, 2001.

20. Pu Y, Wang L, Wu H, Feng Z, Wang Y and Guo C: High MMP-21 expression in metastatic lymph nodes predicts unfavorable overall survival for oral squamous cell carcinoma patients with lymphatic metastasis. Oncol Rep 31: 2644-2650, 2014.

21. Ye F, Chen C, Qin J, Liu J and Zheng C: Genetic profiling reveals an alarming rate of cross-contamination among human cell lines used in China. FASEB J 29: 4268-4272, 2015.

22. Zhou X, Liu S, Cai G, Kong L, Zhang T, Ren Y, Wu Y, Mei M, Zhang L and Wang X: Long Non Coding RNA MALAT1 promotes tumor growth and metastasis by inducing epithelial-mesenchymal transition in oral squamous cell carcinoma. Sci Rep 5: 15972, 2015.

23. Diepenbruck M and Christofori G: Epithelial-mesenchymal transition (EMT) and metastasis: Yes, no, maybe? Curr Opin Cell Biol 43: 7-13, 2016.

24. Davis FM, Stewart TA, Thompson EW and Monteith GR: Targeting EMT in cancer: Opportunities for pharmacological intervention. Trends Pharmacol Sci 35: 479-488, 2014.

25. da Silva SD, Morand GB, Alobaid FA, Hier MP, Mlynarek AM, Alaoui-Jamali MA and Kowalski LP: Epithelial-mesenchymal transition (EMT) markers have prognostic impact in multiple primary oral squamous cell carcinoma. Clin Exp Metastas 32: 55-63, 2015.

26. Giroldi LA, Bringuier PP, de Weijert M, Jansen C, van Bokhoven A and Schalken JA: Role of $\mathrm{E}$ boxes in the repression of E-cadherin expression. Biochem Bioph Res Commun 241: 453-458, 1997.

27. Wang Y, Shi J, Chai K, Ying X and Zhou BP: The role of Snail in EMT and tumorigenesis. Curr Cancer Drug Tar 13: 963-972, 2013.

28. Li YY, Zhou CX and Gao Y: Snail regulates the motility of oral cancer cells via RhoA/Cdc42/p-ERM pathway. Biochem Bioph Res Commun 452: 490-496, 2014.

29. Zhu LF, Hu Y, Yang CC, Xu XH, Ning TY, Wang ZL, Ye JH and Liu LK: Snail overexpression induces an epithelial to mesenchymal transition and cancer stem cell-like properties in SCC9 cells. Lab Invest 92: 744-752, 2012.

30. Gonzalez DM and Medici D: Signaling mechanisms of the epithelial-mesenchymal transition. Sci Signal 7: re8, 2014.

31. Wang ZW, Li YW, Kong DJ and Sarkar FH: The role of Notch signaling pathway in epithelial-mesenchymal transition (EMT) during development and tumor aggressiveness. Curr Drug Targets 11: 745-751, 2010.

32. Li YM, Ma J, Qian XJ, Wu Q, Xia J, Miele L, Sarkar FH and Wang ZW: Regulation of EMT by Notch signaling pathway in tumor progression. Curr Cancer Drug Tar 13: 957-962, 2013.

33. Li LC, Peng Y, Liu YM, Wang LL and Wu XL: Gastric cancer cell growth and epithelial-mesenchymal transition are inhibited by $\gamma$-secretase inhibitor DAPT. Oncol Lett 7: 2160-2164, 2014. 